Supplementary materials for

\title{
Chemico-proteomics reveal the enhancement of salt tolerance in an invasive plant species via $\mathrm{H}_{2} \mathrm{~S}$ signaling
}

Jiabing $\mathrm{Li}^{1,2}$, Zixian $\mathrm{Yu}^{1,2 \#}$, Simeon $\mathrm{Choo}^{3 \#}$, Jingying Zhao ${ }^{1,2 \#}$, Zhezhe Wang ${ }^{4,5^{*}}$, Rongrong $\mathrm{Xie}^{1,2^{*}}$

1. College of Environmental Science and Engineering, Fujian Normal University, Fuzhou 350007, China;

2. Key Laboratory of Pollution Control and Resource Recycling of Fujian Province, Fujian Normal University, Fuzhou 350007, China;

3. Department of Biological Oceanography, Leibniz Institute for Baltic Sea Research, Warnemuende D-18119 Rostock, Germany

4. College of Physics and Energy, Fujian Normal University, Fuzhou 350117, China

5. Fujian Provincial Key Laboratory of Quantum Manipulation and New Energy Materials, Fuzhou 350117, China

\# The authors contributed equally to this work.

*Correspondence to

Rongrong Xie

Address: 8 Shangsan Rd, Fuzhou, 350007, China

Email: xierr1118@163.com

Zhezhe Wang

Address: 8 Shangsan Rd, Fuzhou, 350007, China

Email: zzwang@,fjnu.edu.cn.

\section{Contents}

Supplementary Text 
Supplementary Figure S1

Supplementary Figure S2

Supplementary Figure S3

Supplementary Figure S4

Supplementary Figure S5

Supplementary Figure S6

Supplementary Table S1 


\section{Supplementary Text}

\section{(1) Measurement of lipid peroxidation}

A total of $1 \mathrm{~g}$ of leaves was homogenized in $10 \mathrm{~mL}$ of $10 \%$ trichloroacetic acid (TCA). The homogenate was then centrifuged at $10000 \times \mathrm{g}$ for $20 \mathrm{~min}$. A $1-\mathrm{mL}$ aliquot of the supernatant was added to $4 \mathrm{~mL}$ of $0.6 \%$ thiobarbituric acid (TBA) in $20 \%$ TCA. The mixture was heated to $95{ }^{\circ} \mathrm{C}$, maintained at that temperature for $30 \mathrm{~min}$, and then quickly cooled in an ice bath. After centrifugation at $10000 \times \mathrm{g}$ for $10 \mathrm{~min}$, a $1-\mathrm{mL}$ aliquot of the supernatant was added to $3 \mathrm{~mL}$ of $20 \%$ TCA containing $0.5 \%(\mathrm{w} / \mathrm{v})$ thiobarbituric acid (TBA). The mixture was incubated at $95{ }^{\circ} \mathrm{C}$ for $30 \mathrm{~min}$ and the reaction was stopped by quickly placing the sample in an ice bath. The cooled mixture was centrifuged at $10000 \times \mathrm{g}$ for $10 \mathrm{~min}$ at room temperature and the absorbance volume of the supernatant was read at 532 and $600 \mathrm{~nm}$.

\section{(2) L-CDs enzyme activity measurement}

L-CD activity was determined by measuring the release of $\mathrm{H}_{2} \mathrm{~S}$ from L-cysteine as described with minor modifications. The assay contained a total volume of $1 \mathrm{~mL}: 0.1$ $\mathrm{mL}$ of $10 \mathrm{mM}$ L-cysteine, $2.5 \mathrm{mM}$ DTT, $0.8 \mathrm{~mL}$ of $100 \mathrm{mM}$ Tris-HCl buffer containing $2.5 \mathrm{mM}$ DTT, $\mathrm{pH} 9.0$, and $0.1 \mathrm{~mL}$ of protein solution. The reaction was initiated by adding L-cysteine. After incubation for 60 min at $37{ }^{\circ} \mathrm{C}$, the reaction was terminated by adding $0.1 \mathrm{~mL}$ of $30 \mathrm{mM} \mathrm{FeCl}_{3}$ dissolved in $1.2 \mathrm{~N} \mathrm{HCl}$ and $0.1 \mathrm{~mL}$ of $20 \mathrm{mM} \mathrm{N}, \mathrm{N}-$ dimethyl-p-phenylenediamine dihydrochloride dissolved in $7.2 \mathrm{M} \mathrm{HCl}$. The formation of methylene blue was determined at $670 \mathrm{~nm}$, and known concentrations of $\mathrm{Na}_{2} \mathrm{~S}$ were used in the calibration curve. One unit of enzyme activity for L-CD is defined as the 
amount of enzyme that produces $1 \mu \mathrm{mol} \min ^{-1} \mathrm{H}_{2} \mathrm{~S}$ under the stated assay conditions and is expressed as $\mathrm{U} \mathrm{g}^{-1} \mathrm{FW}$.

\section{(3) Protein extraction and 2D electrophoresis}

Approximately 10 to $20 \mathrm{~g}$ of leaves was ground in liquid nitrogen and the total soluble protein was extracted at $4{ }^{\circ} \mathrm{C}$ in $5 \mathrm{~mL}$ of $50 \mathrm{mM}$ Tris- $\mathrm{HCl}$ buffer $(\mathrm{pH} 7.5)$ containing $20 \mathrm{mM} \mathrm{KCl}, 13 \mathrm{mM}$ DTT, $2 \%$ (v/v) NP-40, $150 \mathrm{mM}$ PMSF, and $1 \%$ (w/v) PVPP. The homogenates were centrifuged $\left(12000 \mathrm{~g}, 15 \mathrm{~min}, 4^{\circ} \mathrm{C}\right)$ and the supernatants were added to five volumes of acetone containing $10 \%(\mathrm{w} / \mathrm{v})$ TCA and $1 \%(\mathrm{w} / \mathrm{v})$ DTT. The samples were maintained at $-20{ }^{\circ} \mathrm{C}$ for $4 \mathrm{~h}$ and then centrifuged $(25000 \times \mathrm{g}, 30 \mathrm{~min}$, $\left.4{ }^{\circ} \mathrm{C}\right)$. The resulting pellets were washed with acetone containing $1 \%(\mathrm{w} / \mathrm{v})$ DTT at $20{ }^{\circ} \mathrm{C}$ for $1 \mathrm{~h}$ and then centrifuged at $12000 \times \mathrm{g}$, and this wash step was repeated. The pellets were vacuum-dried and dissolved in $8 \mathrm{M}$ urea, $20 \mathrm{mM} \mathrm{DTT}, 4 \%(\mathrm{w} / \mathrm{v})$ CHAPS, and $2 \%(\mathrm{w} / \mathrm{v})$ ampholyte $(\mathrm{pH} 4-7)$, and then centrifuged at $30000 \times \mathrm{g}$ for $20 \mathrm{~min}$ at

$4{ }^{\circ} \mathrm{C}$. The supernatant was subjected to a secondary clarifying centrifugation at 30 $000 \times \mathrm{g}$ for $20 \mathrm{~min}$, and the resulting supernatant was used to assay the protein content and for 2-DE. The protein concentration was determined by the Bradford method, using bovine serum albumin as the standard. ${ }^{41}$ Each experiment was repeated three times. Extracted proteins were first separated by isoelectric focusing (IEF) using gel strips to form an immobilized nonlinear $\mathrm{pH}$ gradient from 4 to 7 (Immobiline DryStrip, $\mathrm{pH} 4-7$ NL, $17 \mathrm{~cm}$; Bio-Rad) and then by SDS-PAGE using $12.5 \%$ polyacrylamide gels. The strips were rehydrated for $16 \mathrm{~h}$ in $450 \mu \mathrm{L}$ of dehydration buffer containing $800 \mu \mathrm{g}$ of total proteins and a trace of bromophenol blue. The strips were then focused at $64 \mathrm{kV} / \mathrm{h}$ 
and $20^{\circ} \mathrm{C}$ using the PROTEAN IEF System (Bio-Rad). After IEF, the strips were equilibrated for $15 \mathrm{~min}$ in equilibration buffer (6 M urea, $0.375 \mathrm{M}$ Tris, $\mathrm{pH} 8.8,2 \%$ (w/v) SDS, $20 \%(\mathrm{v} / \mathrm{v})$ glycerol, and $2 \%(\mathrm{w} / \mathrm{v})$ DTT). For 2D SDS-PAGE, the strips were placed on $12.5 \%$ (w/v) SDS-PAGE gels. Gel electrophoresis was performed at $25 \mathrm{~mA}$ for $5 \mathrm{~h}$. The gels were stained using the colloidal CBB staining method. After staining, the gels were scanned using a GS-800 Calibrated Densitometer (Bio-Rad) and analyzed with PDQUEST software (Bio-Rad), using 10-fold over background as the minimum criterion for the presence/absence of signal for the guided protein spot detection method. Parameters were optimized as follows: saliency, 2.0; partial threshold, 4; and minimum area, 50. Three well-separated gels of the treatment group were used to create the replicate groups. Spots were considered reproducible when they were well-resolved in the three biological replicates. For each matched spot, a measurement was taken for each biological replicate. The normalized volume of each spot was assumed to represent its expression abundance. A criterion of at least 1.6-fold and $p<0.05$ was used to define significant differences when comparing spot size between groups with one-way ANOVA using the SPSS v. 19 package (http://spss.en.softonic.com/). To compensate for subtle differences in sample loading or gel staining/destaining during individual replicate experiments, the volume of each spot was normalized against the control gel. Each experiment was repeated three times. 


\section{(4) In-gel digestion}

Firstly, individual spots of interest were excised from the 2DE gels using sterile tips and placed in $1.5 \mathrm{~mL}$ sterile tubes. Then, each polyacrylamide spot was destained with $50 \mathrm{mM} \mathrm{NH}_{4} \mathrm{HCO}_{3}$ for $1 \mathrm{~h}$ at $40{ }^{\circ} \mathrm{C}$, reduced with $10 \mathrm{mM}$ DTT in $100 \mathrm{Mm} \mathrm{NH}_{4} \mathrm{HCO}_{3}$

for $1 \mathrm{~h}$ at $60{ }^{\circ} \mathrm{C}$, and thereafter incubated with $40 \mathrm{mM}$ iodoacetamide in $100 \mathrm{mM}$ $\mathrm{NH}_{4} \mathrm{HCO}_{3}$ for $30 \mathrm{~min}$. The gel pieces were minced and allowed to dry, and then rehydrated in $12.5 \mathrm{ng} / \mu \mathrm{L}$ trypsin (sequencing grade; Roche Diagnostics, Indiana, USA) in $25 \mathrm{mM} \mathrm{NH}_{4} \mathrm{HCO}_{3}$ at $37{ }^{\circ} \mathrm{C}$ overnight. The trypsin peptides were extracted from the gel grains with $0.1 \%$ trifluoroacetic acid in $50 \%$ acetonitrile. This step was performed thrice. Supernatants were concentrated in a SpeedVac Concentrator to approximately $10 \mu \mathrm{L}$ and desalted using Zip-Tips (C18resin, P10; Millipore Corporation, MA, USA). Peptides were eluted from the column with $50 \%$ acetonitrile/ $0.1 \%$ trifluoroacetic acid. The protein spots that changed by more than 1.5 -fold (compared with the sample spots at $4350 \mathrm{~m})$ and passed the one-way ANOVA test $(p<0.05)$ were selected and identified by mass spectrometry (MS) analysis.

\section{(5) MALDI-TOF/TOF analysis and database search}

The MS acquisition and processing parameters were: reflector positive mode and 800-3500 Da acquisition mass range. The laser frequency was $50 \mathrm{~Hz}$ and 700 laser points were collected for each sample signal. For each sample, 3 to 6 ion peaks with signal-to-noise ratios greater than 100 were selected as precursors for secondary MS analysis, and the TOF/TOF signal for each precursor was assessed with 1500 laser points. The primary and secondary MS data were transferred into Microsoft Excel files 
as inputs to search against a NCBI non-redundant database. The search was restricted to viridiplantae (green plants) using the MASCOT search engine (www.matrixscience.com). The search parameters were established as follows: no restriction of protein molecular weight; one missed trypsin cleavage allowed; cysteine treated with iodoacetamide; and oxidation of methionine. The peptide tolerance was $100 \mathrm{ppm}$ and the MS/MS tolerance were $0.25 \mathrm{kD}$. Protein identifications were validated manually, with at least 4 peptides matching. Keratin contamination was removed and the MOWSE score threshold was greater than $60(p<0.05)$. According to the MASCOT probability analysis, only significant hits with the highest protein score and sequence coverage were accepted for the identification of the protein sample. 


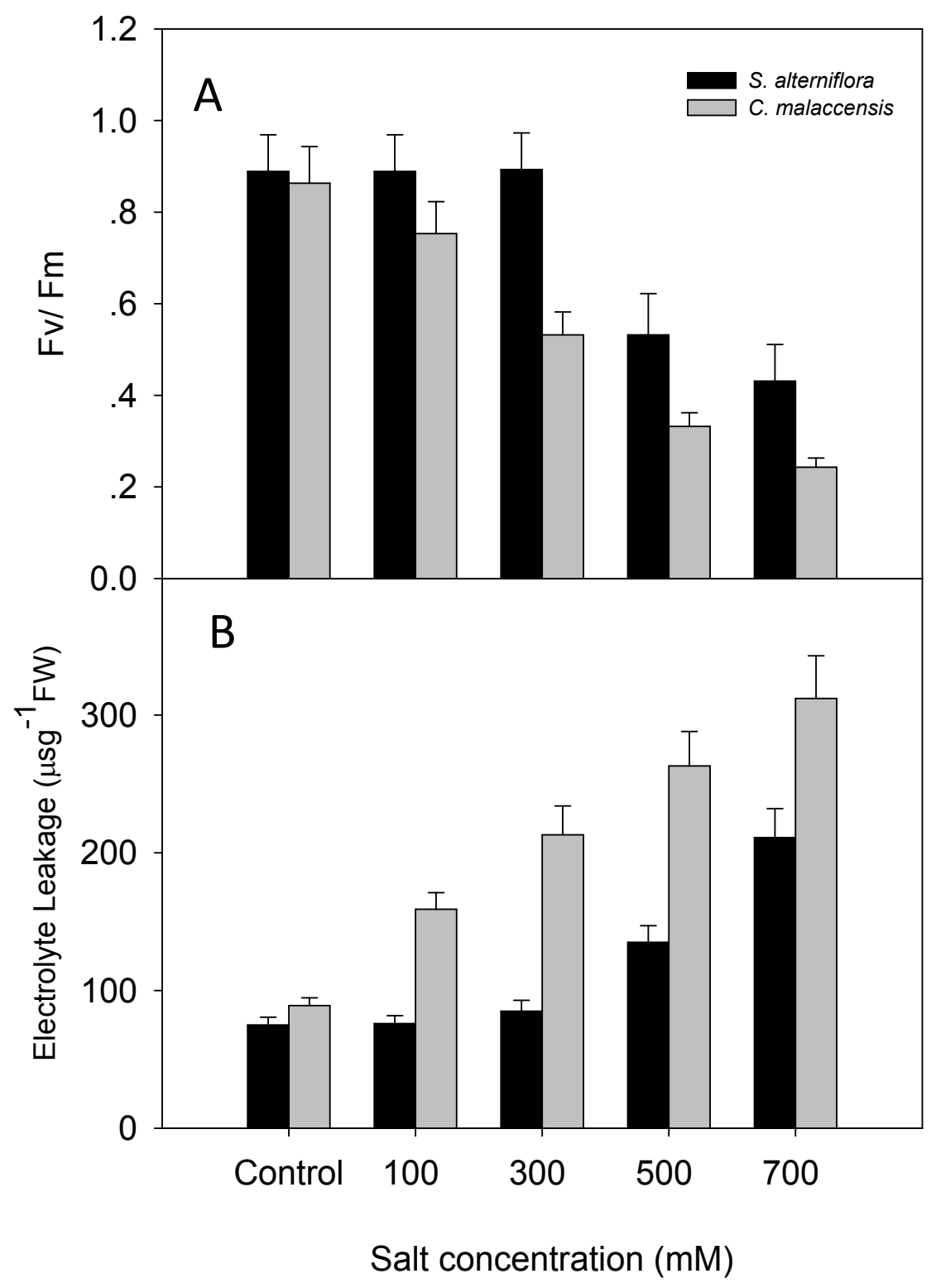

Supplemental Figure S1. Dose effect of salt on photosynthetic Fv/Fm (A) and ion leakage (B) of S. alterniflora.S. alterniflora and Cyperusmalaccensis seedlings were treated with different concentrations of salt for 3 days, and Fv/Fm and ion leakage were measured. 


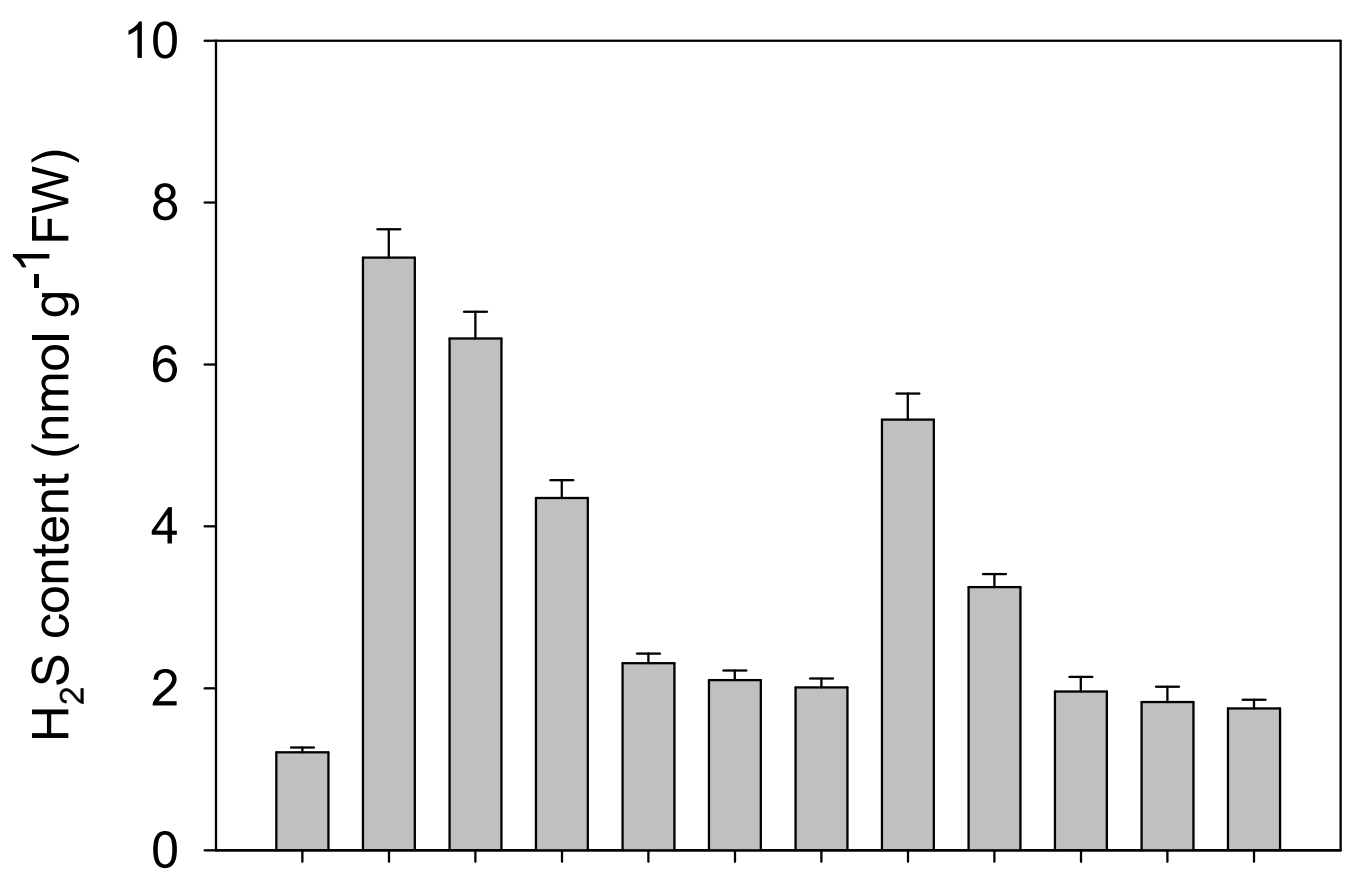

Supplemental Figure S2. Dose effect of PA and HT on salt-induced $\mathrm{H}_{2} \mathrm{~S}$ generation. $S$. alterniflora seedlings were treated with $300 \mathrm{mM} \mathrm{NaCl}$ in addition to different concentrations of PAG or HT for 3 days, and $\mathrm{H}_{2} \mathrm{~S}$ generation was measured. 


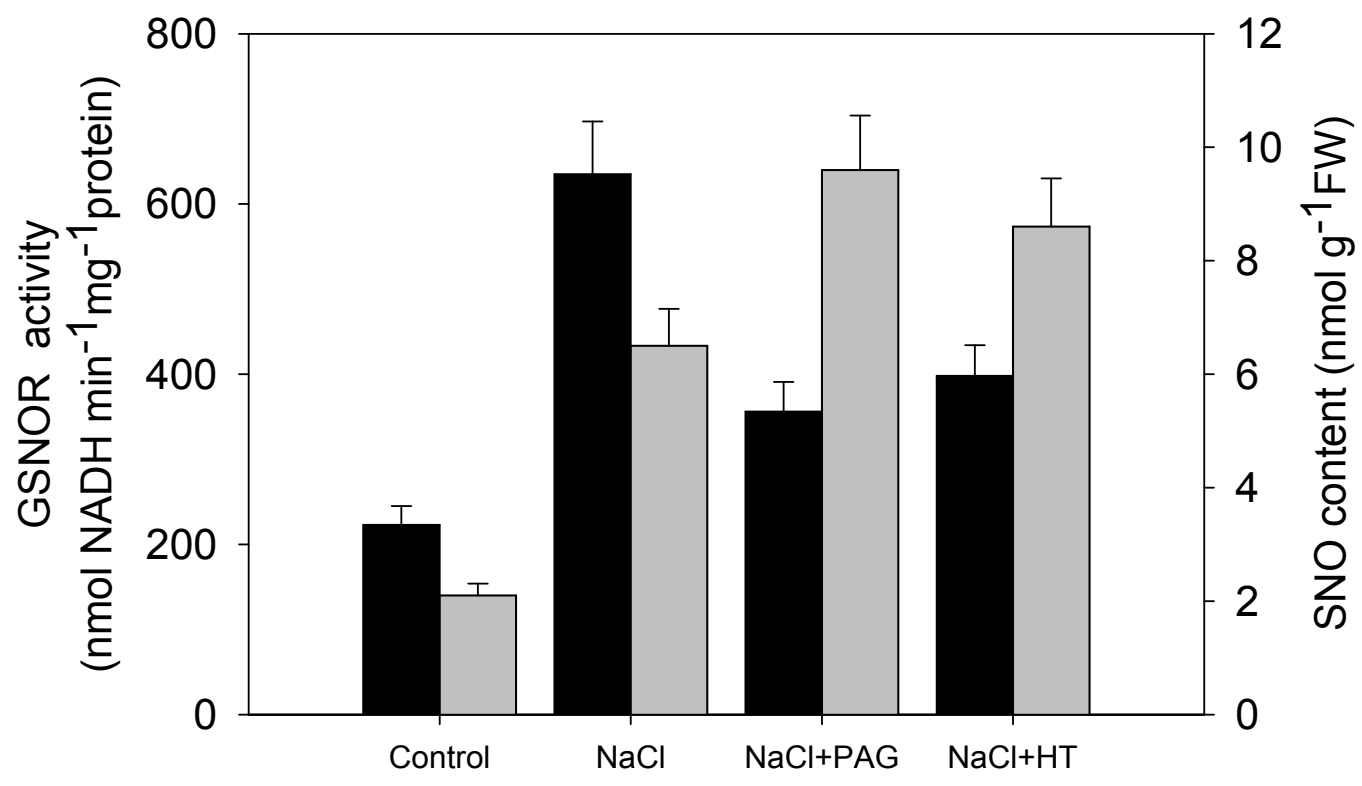

Supplemental Figure S3. Effects of PAG $(2 \mathrm{mM})$ and HT $(100 \mu \mathrm{M})$ on GSNOR activity and SNO content under salt stress. S. alterniflora seedlings were treated with $300 \mathrm{mM} \mathrm{NaCl}$ for 3 days with or without PAG and HT treatment, the GSNOR activity and SNO content were measured. 


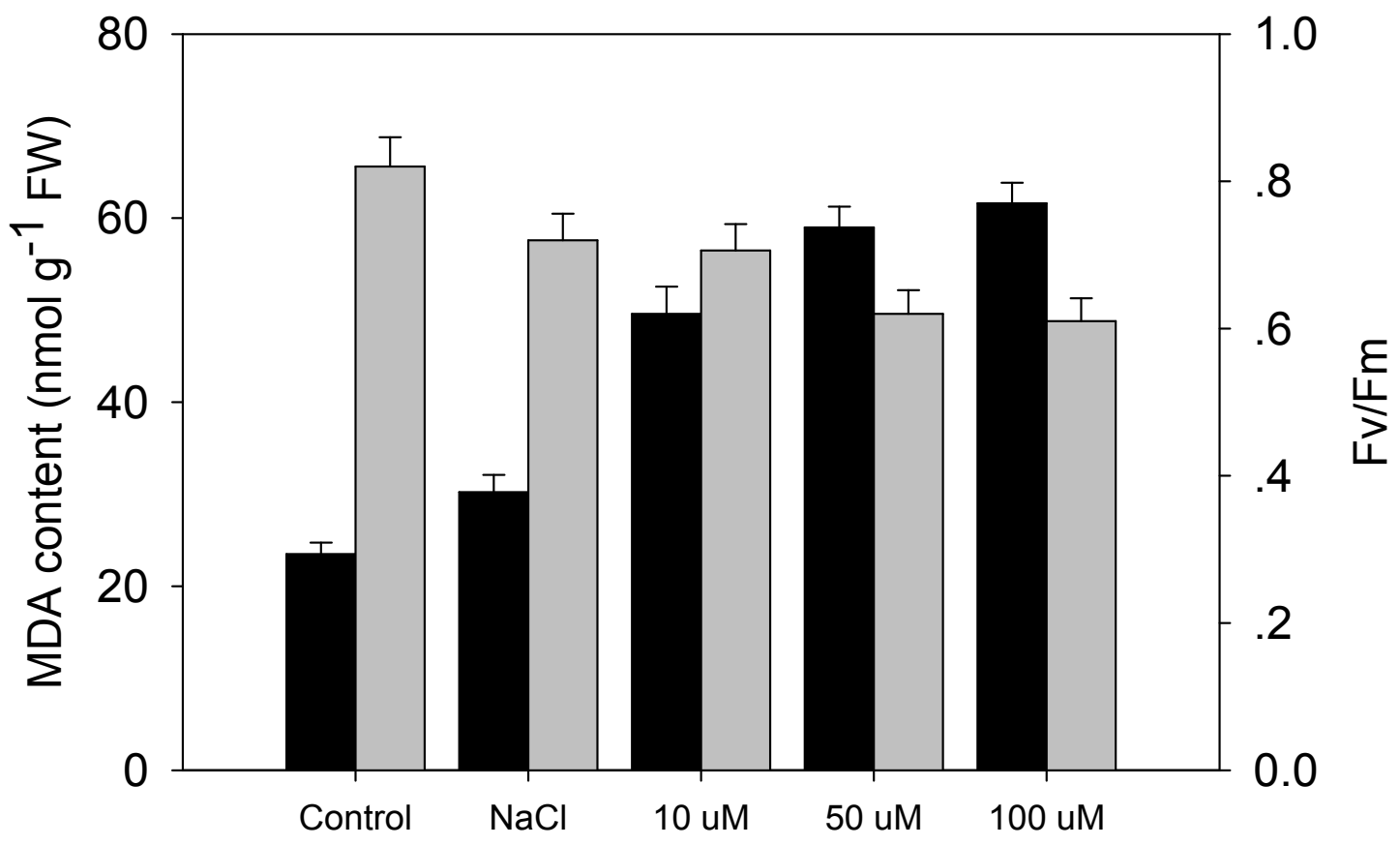

Supplemental Figure S4. Effect of DA on the MDA content and Fv/Fm ratio was evaluated in

S. alterniflora leaves after exposure to salt stress. S. alterniflora seedlings were treated with $300 \mathrm{mM} \mathrm{NaCl}$ and then with different concentrations of DA for 3 days, and the MDA content and $\mathrm{Fv} / \mathrm{Fm}$ ratio were measured. 


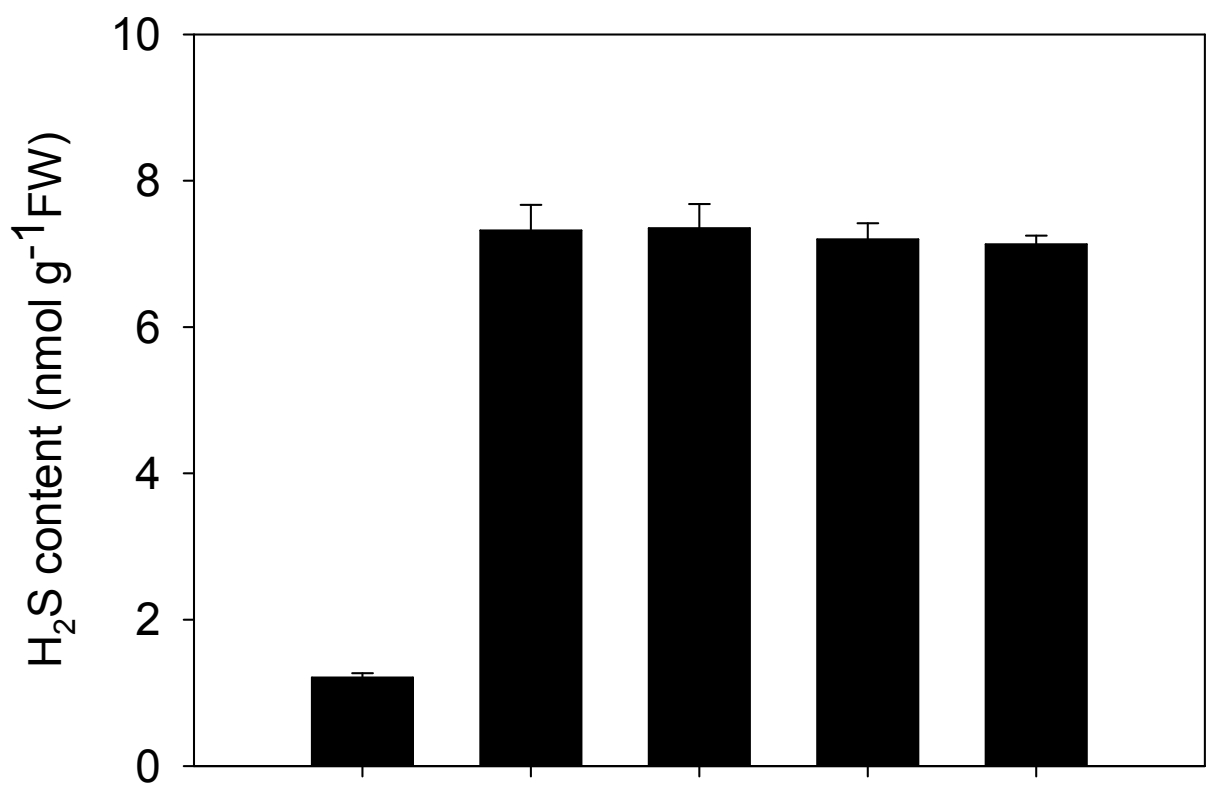

Supplemental Figure S5. Effect of DA on $\mathrm{H}_{2} \mathrm{~S}$ generation was evaluated in S. alterniflora leaves after exposure to salt stress. S. alterniflora seedlings were treated with $300 \mathrm{mM} \mathrm{NaCl}$ and then different concentrations of DA for 3 days, and $\mathrm{H}_{2} \mathrm{~S}$ generation was measured. 

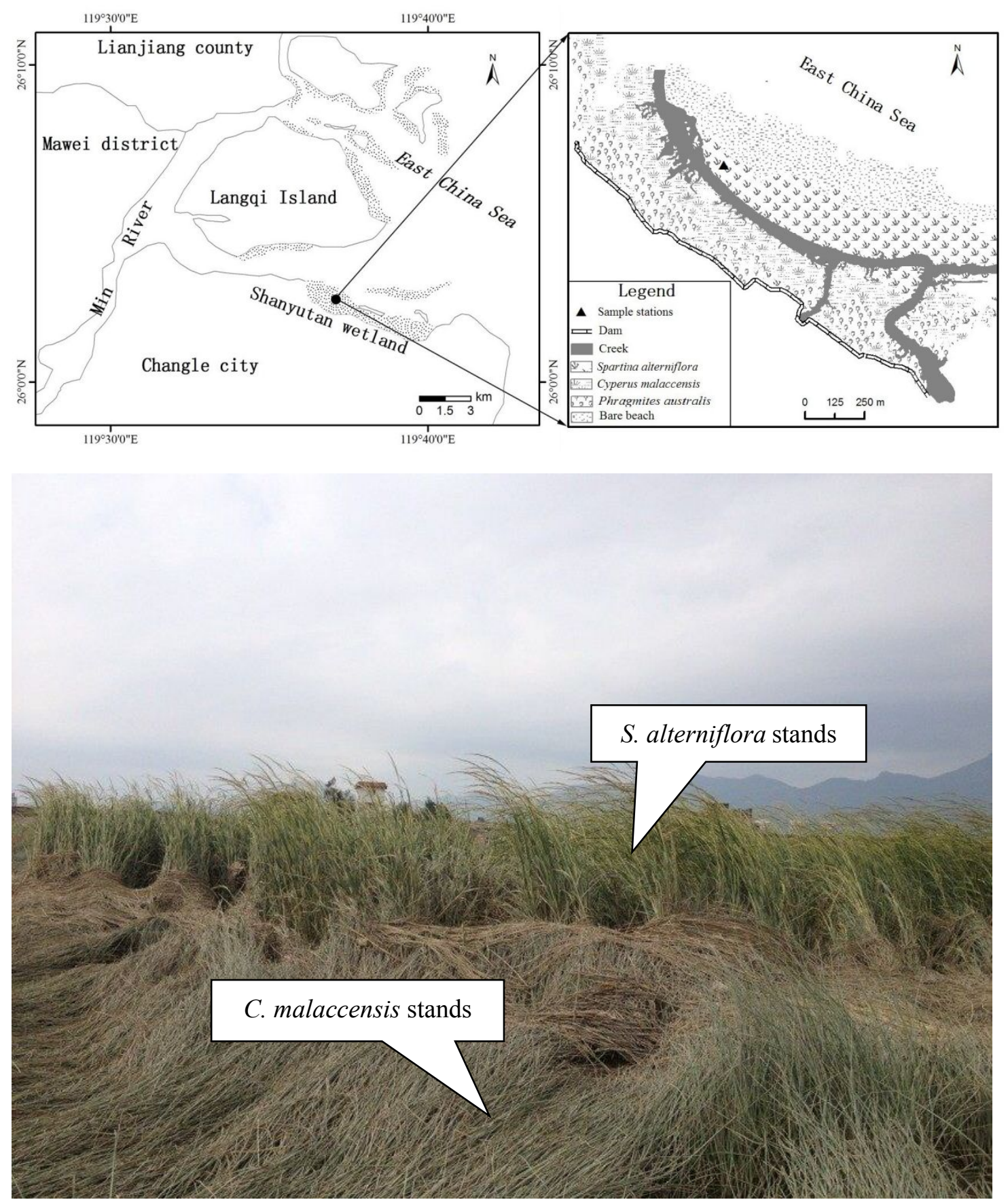

Supplemental Figure S6. Detailed information on sample site (The photo was taken by

Jiabing Li)

Within this area, there is a mosaic vegetation landscape dominated by $C$. malaccensis and $S$. alterniflora stands. We selected a C. malaccensis stand and a $S$. alterniflora stand for our analysis. These two stands were adjacent, and were monocultures with clear boundaries between them. Aside from C. malaccensis and S. alterniflora, the selected area was otherwise homogeneous. 
Supplemental Table S1. Identification of S. alternifloraproteins that are differentially expressed by more than 1.5 -fold or less than 0.6 -fold after salt treatment using MALDI-MS/MS analysis.

\begin{tabular}{|c|c|c|c|c|c|c|c|c|c|c|c|}
\hline $\begin{array}{l}\text { Spot } \\
\text { NO. }\end{array}$ & NCBI Accession No. ${ }^{a}$ & Protein Name & $\begin{array}{l}\text { Exp.Mw / } \\
\text { pI }^{\mathrm{b}}\end{array}$ & $\begin{array}{l}\text { Theo. Mw } \\
\qquad / \mathrm{pI}^{\mathrm{c}}\end{array}$ & $\begin{array}{l}\text { Score }^{\mathrm{d}} / \mathrm{Cov}- \\
\text { erage }(\%)^{\mathrm{e}}\end{array}$ & $\begin{array}{l}1 \mathrm{~d} \\
/ \mathrm{CK}\end{array}$ & $\begin{array}{l}\mathrm{p} \\
\text { value } \\
\mathrm{g}\end{array}$ & $\begin{array}{l}\quad \mathrm{R} \\
3 \mathrm{~d} \\
/ \mathrm{CK}\end{array}$ & $\begin{array}{l}\text { tio }^{\mathrm{f}} \\
\mathrm{p} \\
\text { value } \\
\mathrm{g}\end{array}$ & $\begin{array}{l}5 \mathrm{~d} \\
/ \mathrm{CK}\end{array}$ & $\begin{array}{l}\mathrm{p} \\
\text { value } \\
\mathrm{g}\end{array}$ \\
\hline \multicolumn{12}{|c|}{ Material and energy related } \\
\hline 10 & Putative terpenoid synthase 5 & $\mathrm{O} 22184$ & $74.04 / 5.41$ & $\begin{array}{l}68.48 / \\
5.36\end{array}$ & $121 / 9.44 \%$ & 1.56 & 0.02 & 2.94 & 0.04 & 5.84 & 0.01 \\
\hline 12 & Beta-galactosidase 8 & Q0DGD7 & $81.45 / 5.54$ & $\begin{array}{l}5.56 / \\
73.52\end{array}$ & $101 / 9.20 \%$ & 0.62 & 0.02 & 0.43 & 0.03 & 0.18 & 0.01 \\
\hline 14 & Sucrose synthase & P10691 & $84.49 / 5.75$ & $\begin{array}{l}92.41 / \\
5.83\end{array}$ & $133 / 9.16 \%$ & 2.05 & 0.01 & 3.09 & 0.01 & 5.34 & 0.03 \\
\hline 24 & Monosaccharide-sensing protein 3 & Q9SD00 & $73.74 / 5.23$ & $\begin{array}{l}78.82 / \\
5.25\end{array}$ & $105 / 6.47 \%$ & 2.02 & 0.04 & 3.1 & 0.03 & 3.68 & 0.02 \\
\hline 26 & (E)-beta-farnesene synthase & C7E5V7 & $64.89 / 5.32$ & $\begin{array}{l}61.36 / \\
5.25\end{array}$ & $133 / 6.65 \%$ & 0.71 & 0.04 & 0.58 & 0.01 & 0.51 & 0.01 \\
\hline 30 & $\begin{array}{l}\text { Soluble starch synthase } 1 \text {, } \\
\text { chloroplastic/amyloplastic. }\end{array}$ & A2Y9M4 & $64.28 / 5.69$ & $\begin{array}{l}59.23 / \\
5.56\end{array}$ & $133 / 11.20 \%$ & 2.8 & 0.03 & 3.1 & 0.02 & 3.76 & 0 \\
\hline 31 & Santalene synthase & E3W203 & $70.34 / 5.80$ & $\begin{array}{l}65.26 / \\
5.75\end{array}$ & $159 / 11.40 \%$ & 1.43 & 0.03 & 2.01 & 0.02 & 3.34 & 0.02 \\
\hline 34 & Beta-fructofuranosidase & A2YZ01 & $67.07 / 5.87$ & $\begin{array}{l}63.09 / \\
5.89\end{array}$ & $144 / 9.24 \%$ & 0.62 & 0.01 & 0.47 & 0 & 0.32 & 0.01 \\
\hline 35 & Phenylalanine ammonia-lyase & A2X7F7 & $71.20 / 5.93$ & $\begin{array}{l}76.93 / \\
5 / 97\end{array}$ & $98 / 7.03 \%$ & 1.12 & 0.03 & 0.56 & 0.01 & 0.43 & 0.04 \\
\hline
\end{tabular}




\begin{tabular}{|c|c|c|c|c|c|c|c|c|c|c|c|}
\hline Spot & NCBI Accession No. ${ }^{\mathrm{a}}$ & Protein Name & Exp.Mw / & Theo. Mw & Score $^{d} /$ Cov- & & & & $\operatorname{tio}^{\mathrm{f}}$ & & \\
\hline NO. & & & $\mathrm{pI}^{\mathrm{b}}$ & $/ \mathrm{pI}^{\mathrm{c}}$ & erage $(\%)^{\mathrm{e}}$ & $\begin{array}{l}1 \mathrm{~d} \\
/ \mathrm{CK}\end{array}$ & $\begin{array}{l}\mathrm{p} \\
\text { value } \\
\mathrm{g}\end{array}$ & $\begin{array}{l}3 \mathrm{~d} \\
/ \mathrm{CK}\end{array}$ & $\begin{array}{l}\mathrm{p} \\
\text { value } \\
\mathrm{g}\end{array}$ & $\begin{array}{l}5 \mathrm{~d} \\
/ \mathrm{CK}\end{array}$ & $\begin{array}{l}\mathrm{p} \\
\text { value } \\
\mathrm{g}\end{array}$ \\
\hline 42 & Beta-amylase & P10537 & $50.94 / 5.11$ & $\begin{array}{l}55.94 / \\
5.17\end{array}$ & $167 / 12.20 \%$ & 1.3 & 0.01 & 2.33 & 0.04 & 4.34 & 0.01 \\
\hline 43 & Alpha-amylase isozyme $2 \mathrm{~A}$ & A2YGY2 & $47.09 / 5.17$ & $\begin{array}{l}46.55 / \\
5.11\end{array}$ & $199 / 12.78 \%$ & 1.24 & 0.02 & 2.07 & 0.02 & 4.5 & 0.04 \\
\hline 53 & Inositol-3-phosphate synthase & Q41107 & $52.77 / 5.39$ & $\begin{array}{l}56.52 / \\
5.30\end{array}$ & $175 / 14.42 \%$ & 0.78 & 0.01 & 0.32 & 0 & 0.21 & 0.03 \\
\hline 65 & $\begin{array}{l}\text { Putative D-cysteine desulfhydrase } \\
2, \text { mitochondrial }\end{array}$ & B9EYZ1 & $40.27 / 6.46$ & $40.96 / 6.4$ & $192 / 22.22 \%$ & 2.72 & 0.04 & 3.88 & 0 & 4.67 & 0.01 \\
\hline 79 & $\begin{array}{l}\text { Cystathionine gamma-lyase / } \\
\text { cysteine synthase }\end{array}$ & A0A178UEA4 & $32.22 / 5.60$ & $\begin{array}{l}34.32 / \\
5.61\end{array}$ & $233 / 20.40 \%$ & 1.65 & 0.02 & 2.01 & 0.01 & 2.23 & 0.03 \\
\hline 66 & Methylecgonone reductase & E7C196 & $40.78 / 6.51$ & $\begin{array}{l}36.90 / \\
6.33\end{array}$ & $183 / 14.71 \%$ & 0.73 & 0.02 & 0.55 & 0.02 & 0.33 & 0.01 \\
\hline 69 & GSNOR & A2XAZ3 & $40.01 / 6.80$ & $\begin{array}{l}40.79 / \\
6.79\end{array}$ & $184 / 13.14 \%$ & 2.69 & 0.03 & 1.84 & 0.03 & 1.35 & 0.03 \\
\hline 73 & $\begin{array}{l}\text { Probable 6- } \\
\text { phosphogluconolactonase } 1\end{array}$ & A2XI04 & $30.57 / 4.91$ & $\begin{array}{l}33.21 / \\
4.95\end{array}$ & $155 / 15.60 \%$ & 1.29 & 0.03 & 2.7 & 0.04 & 4.34 & 0.03 \\
\hline 78 & $\begin{array}{l}\text { Fructose-1,6-bisphosphatase, } \\
\text { cytosolic }\end{array}$ & A2WXB2 & $35.51 / 5.52$ & $\begin{array}{l}37.03 / \\
5.55\end{array}$ & $231 / 20.87 \%$ & 1.4 & 0.01 & 2.92 & 0.04 & 3.38 & 0.03 \\
\hline 82 & Metalloendoproteinase 3-MMP & Q5XF51 & $22.11 / 4.91$ & $\begin{array}{l}22.07 / \\
5.01\end{array}$ & $123 / 12.02 \%$ & 0.63 & 0.02 & 0.44 & 0.02 & 0.33 & 0.02 \\
\hline 83 & Ferritin-3, chloroplastic & O65100 & $25.60 / 5.14$ & $\begin{array}{l}23.12 / \\
5.12\end{array}$ & $284 / 34.48 \%$ & 0.73 & 0.02 & 0.61 & 0.01 & 0.24 & 0.01 \\
\hline
\end{tabular}




\begin{tabular}{|c|c|c|c|c|c|c|c|c|c|c|c|}
\hline Spot & NCBI Accession No. ${ }^{a}$ & Protein Name & Exp.Mw / & Theo. Mw & Score $^{\mathrm{d}} /$ Cov- & & & & $\operatorname{tio}^{\text {f }}$ & & \\
\hline NO. & & & $\mathrm{pI}^{\mathrm{b}}$ & $/ \mathrm{pI}^{\mathrm{c}}$ & erage $(\%)^{\mathrm{e}}$ & $\begin{array}{l}1 \mathrm{~d} \\
/ \mathrm{CK}\end{array}$ & $\begin{array}{l}\mathrm{p} \\
\text { value } \\
\mathrm{g}\end{array}$ & $\begin{array}{l}3 \mathrm{~d} \\
/ \mathrm{CK}\end{array}$ & $\begin{array}{l}\mathrm{p} \\
\text { value } \\
\mathrm{g}\end{array}$ & $\begin{array}{l}5 \mathrm{~d} \\
/ \mathrm{CK}\end{array}$ & $\begin{array}{l}\mathrm{p} \\
\text { value } \\
\mathrm{g}\end{array}$ \\
\hline 90 & Soluble inorganic pyrophosphatase & A2X8Q3 & $26.12 / 5.65$ & $\begin{array}{l}24.16 / \\
5.56\end{array}$ & $289 / 28.44 \%$ & 0.63 & 0.04 & 0.47 & 0.02 & 0.33 & 0.01 \\
\hline \multicolumn{12}{|c|}{ Hormone metabolism and responsible proteins } \\
\hline 49 & Ninja-family protein 8 & B6TCE8 & $47.54 / 5.31$ & $\begin{array}{l}49.60 / \\
5.45\end{array}$ & $112 / 9.91 \%$ & 0.79 & 0.02 & 0.46 & 0.02 & 0.21 & 0.04 \\
\hline 62 & Gibberellin 20 oxidase 1-B & O04706 & $44.38 / 6.21$ & $\begin{array}{l}40.73 / \\
6.19\end{array}$ & $143 / 11.62 \%$ & 1.75 & 0.03 & 2.46 & 0.01 & 1.32 & 0.01 \\
\hline 63 & S-adenosylmethionine synthase 1 & A7PQS0 & $45.03 / 6.26$ & $\begin{array}{l}42.73 / \\
6.19\end{array}$ & $135 / 11.60 \%$ & 0.53 & 0.01 & 0.51 & 0.04 & 0.44 & 0.03 \\
\hline 109 & Auxin-responsive protein SAUR40 & O64538 & $15.39 / 6.27$ & $\begin{array}{l}14.60 / \\
6.19\end{array}$ & $204 / 28.10 \%$ & 1.46 & 0.02 & 1.91 & 0.03 & 2.95 & 0.04 \\
\hline \multicolumn{12}{|c|}{ Epigenetic related protein } \\
\hline 2 & $\begin{array}{l}\text { Isoform } 5 \text { of Nuclear poly(A) } \\
\text { polymerase }\end{array}$ & $\mathrm{O} 82312$ & $92.48 / 5.15$ & $90.9 / 5.43$ & $154 / 8.97 \%$ & 0.73 & 0.04 & 0.21 & 0.04 & 0.19 & 0.03 \\
\hline 5 & Poly [ADP-ribose] polymerase & Q9FK91 & $83.25 / 5.28$ & $\begin{array}{l}91.40 / \\
5.12\end{array}$ & $122 / 7.88 \%$ & 0.55 & 0.02 & 0.45 & 0.02 & 0.33 & 0.03 \\
\hline 6 & SWI/SNF complex subunit SWI3C & Q9XI07 & $84.11 / 5.30$ & $88.25 / 5.14$ & $98 / 9.99 \%$ & 0.65 & 0.03 & 0.42 & 0.01 & 0.38 & 0.04 \\
\hline 18 & $\begin{array}{l}\text { ADP-ribosylation factor GTPase- } \\
\text { activating protein AGD2 }\end{array}$ & Q9C6C3 & $84.39 / 6.25$ & $\begin{array}{l}87.80 / \\
6.22\end{array}$ & $104 / 9.89 \%$ & 0.71 & 0.01 & 0.21 & 0.04 & 0.1 & 0.01 \\
\hline 20 & Probable DNA helicase MCM8 & B8AZ14 & $83.25 / 6.48$ & $\begin{array}{l}86.27 / \\
6.50\end{array}$ & $115 / 6.10 \%$ & 1.65 & 0 & 1.96 & 0.04 & 1.34 & 0.03 \\
\hline
\end{tabular}




\begin{tabular}{|c|c|c|c|c|c|c|c|c|c|c|c|}
\hline Spot & NCBI Accession No. ${ }^{a}$ & Protein Name & Exp.Mw / & Theo. Mw & Score $^{\mathrm{d} / \text { Cov- }}$ & & & & tio $^{f}$ & & \\
\hline NO. & & & $\mathrm{pI}^{\mathrm{b}}$ & $/ \mathrm{pI}^{\mathrm{c}}$ & erage $(\%)^{\mathrm{e}}$ & $\begin{array}{l}1 \mathrm{~d} \\
/ \mathrm{CK}\end{array}$ & $\begin{array}{l}\mathrm{p} \\
\text { value } \\
\mathrm{g}\end{array}$ & $\begin{array}{l}3 \mathrm{~d} \\
/ \mathrm{CK}\end{array}$ & $\begin{array}{l}\mathrm{p} \\
\text { value } \\
\mathrm{g}\end{array}$ & $\begin{array}{l}5 \mathrm{~d} \\
/ \mathrm{CK}\end{array}$ & $\begin{array}{l}\mathrm{p} \\
\text { value } \\
\mathrm{g}\end{array}$ \\
\hline 27 & $\begin{array}{l}\text { U4/U6 small nuclear } \\
\text { ribonucleoprotein PRP4-like } \\
\text { protein }\end{array}$ & $\mathrm{O} 22212$ & $63.73 / 5.41$ & $61.84 / 5.45$ & $97 / 8.33 \%$ & 1.02 & 0 & 1.68 & 0 & 2.23 & 0.02 \\
\hline 76 & PHD finger protein ALFIN-LIKE 5 & A2Y4R8 & $31.04 / 5.34$ & $\begin{array}{l}29.10 / \\
5.44\end{array}$ & $154 / 20.53 \%$ & 1.87 & 0.01 & 1.99 & 0.01 & 1.11 & 0.01 \\
\hline \multicolumn{12}{|c|}{ Cellular structure } \\
\hline 33 & $\begin{array}{l}\text { Light-independent } \\
\text { protochlorophyllide reductase } \\
\text { subunit } \mathrm{N}\end{array}$ & A2T391 & $57.49 / 5.81$ & $\begin{array}{l}51.13 / \\
5.83\end{array}$ & $221 / 17.81 \%$ & 5.89 & 0.02 & 1.65 & 0 & 1.23 & 0.04 \\
\hline 41 & $\begin{array}{l}\text { ATP synthase subunit beta, } \\
\text { chloroplastic }\end{array}$ & O03081 & $53.19 / 5.09$ & $\begin{array}{l}53.04 / \\
5.03\end{array}$ & $156 / 12.38 \%$ & 1.4 & 0.04 & 2.99 & 0 & 3.54 & 0.01 \\
\hline 45 & Cyclin-A3-3 & A0MEB5 & $41.26 / 5.13$ & $\begin{array}{l}38.13 / \\
5.05\end{array}$ & $223 / 24.92 \%$ & 0.82 & 0.02 & 0.56 & 0.04 & 0.18 & 0.03 \\
\hline 81 & $\begin{array}{l}\text { Mitotic spindle checkpoint protein } \\
\text { MAD2 }\end{array}$ & Q9LU93 & $22.09 / 4.84$ & $\begin{array}{l}23.73 / \\
4.84\end{array}$ & $184 / 18.57 \%$ & 0.63 & 0.02 & 0.51 & 0.03 & 0.22 & 0.02 \\
\hline 111 & V-type proton ATPase subunit G1 & O82628 & $12.11 / 5.73$ & $\begin{array}{l}12.39 / \\
5.77\end{array}$ & $223 / 32.14 \%$ & 1.35 & 0.01 & 1.73 & 0.01 & 2.23 & 0.02 \\
\hline 113 & $\begin{array}{l}\text { Late embryogenesis abundant } \\
\text { protein }\end{array}$ & P09443 & $10.33 / 5.35$ & $\begin{array}{l}11.07 / \\
5.49\end{array}$ & $221 / 26.92 \%$ & 1.29 & 0.03 & 1.77 & 0 & 2.11 & 0.02 \\
\hline \multicolumn{12}{|c|}{ Protein kinase } \\
\hline 8 & serine/threonine-protein kinase & A2YMV6-2 & $79.53 / 5.37$ & $\begin{array}{l}74.17 / \\
5.28\end{array}$ & $114 / 9.92 \%$ & 1.32 & 0.04 & 1.69 & 0.02 & 2.32 & 0.02 \\
\hline
\end{tabular}




\begin{tabular}{|c|c|c|c|c|c|c|c|c|c|c|c|}
\hline Spot & NCBI Accession No. ${ }^{\mathrm{a}}$ & Protein Name & Exp.Mw / & Theo. Mw & Score $^{d} /$ Cov- & & & & $\operatorname{tio}^{f}$ & & \\
\hline NO. & & & $\mathrm{pI}^{\mathrm{b}}$ & $/ \mathrm{pI}^{\mathrm{c}}$ & erage $(\%)^{\mathrm{e}}$ & $\begin{array}{l}1 \mathrm{~d} \\
/ \mathrm{CK}\end{array}$ & $\begin{array}{l}\mathrm{p} \\
\text { value } \\
\mathrm{g}\end{array}$ & $\begin{array}{l}3 \mathrm{~d} \\
/ \mathrm{CK}\end{array}$ & $\begin{array}{l}\mathrm{p} \\
\text { value } \\
\mathrm{g}\end{array}$ & $\begin{array}{l}5 \mathrm{~d} \\
/ \mathrm{CK}\end{array}$ & $\begin{array}{l}\mathrm{p} \\
\text { value } \\
\mathrm{g}\end{array}$ \\
\hline 28 & $\begin{array}{l}\text { Mitogen-activated protein kinase } \\
\text { kinase } 3\end{array}$ & O80396 & $63.19 / 5.55$ & $\begin{array}{l}57.53 / \\
5.47\end{array}$ & $154 / 11.91 \%$ & 1.44 & 0.02 & 1.94 & 0.03 & 2.21 & 0.03 \\
\hline 32 & $\begin{array}{l}\text { Serine/threonine protein } \\
\text { phosphatase } 2 \mathrm{~A}\end{array}$ & $\mathrm{~A} 2 \mathrm{X} 2 \mathrm{~K} 3$ & $64.77 / 5.80$ & $\begin{array}{l}59.04 / \\
5.77\end{array}$ & $194 / 12.73 \%$ & 1.12 & 0.04 & 1.52 & 0.03 & 1.65 & 0.02 \\
\hline 74 & Fructokinase-2 & A2YQL4 & $32.38 / 4.94$ & $\begin{array}{l}35.51 / \\
5.01\end{array}$ & $199 / 18.42 \%$ & 0.77 & 0.01 & 0.54 & 0 & 0.41 & 0 \\
\hline \multicolumn{12}{|c|}{ Protein stability } \\
\hline 13 & $\begin{array}{l}\text { ATP-dependent zinc } \\
\text { metalloprotease FTSH } 9\end{array}$ & A2ZVG7 & $84.39 / 5.68$ & $\begin{array}{l}80.62 / \\
5.69\end{array}$ & $98 / 7.14 \%$ & 2.35 & 0.02 & 3.93 & 0.02 & 4.55 & 0.02 \\
\hline 21 & BTB family PROTEIN 1 & B7U179 & $76.87 / 6.41$ & $\begin{array}{l}81.36 / \\
6.35\end{array}$ & $95 / 8.84 \%$ & 0.79 & 0.03 & 0.53 & 0.03 & 0.33 & 0 \\
\hline 44 & Autophagy protein 5 & $\mathrm{~A} 2 \mathrm{X} 052$ & $44.45 / 5.07$ & $\begin{array}{l}42.33 / \\
5.01\end{array}$ & $167 / 18.86 \%$ & 0.98 & 0.03 & 0.76 & 0.01 & 0.45 & 0.02 \\
\hline 50 & Cysteine protease ATG4 & A2Q1V6 & $50.82 / 5.32$ & $\begin{array}{l}53.80 / \\
5.22\end{array}$ & $189 / 17.54 \%$ & 1.12 & 0 & 0.76 & 0.04 & 0.41 & 0.03 \\
\hline 80 & $\begin{array}{l}\text { Ubiquitin-conjugating enzyme E2- } \\
23 \mathrm{kDa}\end{array}$ & P16577 & $21.78 / 4.40$ & $\begin{array}{l}21.12 / \\
4.40\end{array}$ & $223 / 27.66 \%$ & 1.55 & 0.01 & 2.14 & 0 & 2.45 & 0.04 \\
\hline 86 & Proteasome subunit alpha type-2 & A2YVR7 & $23.80 / 5.28$ & $\begin{array}{l}25.84 / \\
5.39\end{array}$ & $299 / 28.45 \%$ & 0.82 & 0.03 & 0.47 & 0 & 0.13 & 0.04 \\
\hline 104 & $\begin{array}{l}\text { Probable E3 ubiquitin-protein } \\
\text { ligase XERICO }\end{array}$ & Q9SI09 & $18.97 / 6.18$ & $\begin{array}{l}17.92 / \\
6.29\end{array}$ & $194 / 18.18 \%$ & 1.43 & 0.02 & 2.21 & 0 & 0.34 & 0.01 \\
\hline
\end{tabular}




\begin{tabular}{|c|c|c|c|c|c|c|c|c|c|c|c|}
\hline \multirow{2}{*}{$\begin{array}{l}\text { Spot } \\
\text { NO. }\end{array}$} & \multirow[t]{2}{*}{ NCBI Accession No. ${ }^{\mathrm{a}}$} & \multirow[t]{2}{*}{ Protein Name } & \multirow{2}{*}{$\begin{array}{c}\text { Exp.Mw / } \\
\mathrm{pI}^{\mathrm{b}}\end{array}$} & \multirow{2}{*}{$\begin{array}{l}\text { Theo. Mw } \\
/ \mathrm{pI}^{\mathrm{c}}\end{array}$} & \multirow{2}{*}{$\begin{array}{l}\text { Score }^{\mathrm{d}} / \mathrm{Cov}- \\
\text { erage }(\%)^{\mathrm{e}}\end{array}$} & \multicolumn{6}{|c|}{ Ratio $^{f}$} \\
\hline & & & & & & $\begin{array}{l}1 \mathrm{~d} \\
/ \mathrm{CK}\end{array}$ & $\begin{array}{l}\mathrm{p} \\
\text { value } \\
\mathrm{g}\end{array}$ & $\begin{array}{l}3 \mathrm{~d} \\
/ \mathrm{CK}\end{array}$ & $\begin{array}{l}\mathrm{p} \\
\text { value } \\
\mathrm{g}\end{array}$ & $\begin{array}{l}5 \mathrm{~d} \\
/ \mathrm{CK}\end{array}$ & $\begin{array}{l}\mathrm{p} \\
\text { value } \\
\mathrm{g}\end{array}$ \\
\hline 117 & $\begin{array}{l}\text { Ubiquitin-40S ribosomal protein } \\
\text { S27a }\end{array}$ & P27923 & $9.30 / 6.53$ & $\begin{array}{l}85.25 / \\
6.55\end{array}$ & $223 / 23.42 \%$ & 1.48 & 0.03 & 1.65 & 0.02 & 2.61 & 0.01 \\
\hline Trans & riptional factors & & & & & & & & & & \\
\hline 11 & Scarecrow-like protein 34 & P0C884 & $74.48 / 5.48$ & $107 / 7.49 \%$ & $97 / 7.49 \%$ & 1.57 & 0.02 & 1.69 & 0.04 & 2.11 & 0.03 \\
\hline 22 & Basic helix-loop-helix protein A & E3SXU4 & $72.78 / 5.13$ & $\begin{array}{l}72.96 / \\
5.00\end{array}$ & $99 / 8.75 \%$ & 0.89 & 0 & 0.73 & 0.01 & 0.42 & 0.01 \\
\hline 23 & Transcription factor MYC2 & Q39204 & $73.75 / 5.20$ & $\begin{array}{l}67.95 / \\
5.25\end{array}$ & $121 / 6.47 \%$ & 0.75 & 0.01 & 0.6 & 0 & 0.41 & 0.02 \\
\hline 25 & $\begin{array}{l}\text { ETHYLENE INSENSITIVE 3-like } \\
3 \text { protein }\end{array}$ & $\mathrm{O} 23116$ & $66.38 / 5.19$ & $\begin{array}{l}64.04 / \\
5.28\end{array}$ & $123 / 9.19 \%$ & 1.38 & 0.03 & 1.62 & 0.02 & 2.1 & 0.04 \\
\hline 36 & GBF-interacting protein 1-like & A4FVR1 & $67.14 / 5.96$ & $\begin{array}{l}61.87 / \\
5.93\end{array}$ & $143 / 10.94 \%$ & 1.57 & 0.02 & 2.17 & 0.04 & 2.54 & 0.04 \\
\hline 83 & AP-3 complex subunit & F4I562 & $48.67 / 5.36$ & $\begin{array}{l}46.31 / \\
5.39\end{array}$ & $184 / 16.82 \%$ & 1.13 & 0.02 & 1.78 & 0.02 & 2.05 & 0.02 \\
\hline 55 & $\begin{array}{l}\text { Zinc finger protein CONSTANS- } \\
\text { LIKE } 6\end{array}$ & Q8LG76 & $48.85 / 5.44$ & $\begin{array}{l}46.06 / \\
5.47\end{array}$ & $149 / 13.80 \%$ & 0.83 & 0.03 & 0.59 & 0 & 0.12 & 0.02 \\
\hline 60 & $\begin{array}{l}\text { Zinc finger } \mathrm{CCCH} \text { domain- } \\
\text { containing protein } 26\end{array}$ & O48772 & $46.04 / 5.96$ & $\begin{array}{l}49.61 / \\
6.00\end{array}$ & $143 / 13.02 \%$ & 0.73 & 0.03 & 0.55 & 0.03 & 0.22 & 0.03 \\
\hline 94 & Transcription repressor OFP11 & $\mathrm{O} 23341$ & $22.49 / 6.26$ & $\begin{array}{l}20.67 / \\
6.30\end{array}$ & $223 / 23.66 \%$ & 1.23 & 0.02 & 1.65 & 0.02 & 2.11 & 0.04 \\
\hline 95 & $\begin{array}{l}\text { Ethylene-responsive transcription } \\
\text { factor } 13\end{array}$ & Q8L9K1 & $23.25 / 6.36$ & $\begin{array}{l}25.35 / \\
6.33\end{array}$ & $198 / 23.04 \%$ & 0.82 & 0 & 0.71 & 0.02 & 0.44 & 0.04 \\
\hline
\end{tabular}




\begin{tabular}{|c|c|c|c|c|c|c|c|c|c|c|c|}
\hline Spot & NCBI Accession No. ${ }^{a}$ & Protein Name & Exp.Mw / & Theo. Mw & Score $^{\mathrm{d} / \text { Cov- }}$ & & & & $\operatorname{tio}^{f}$ & & \\
\hline NO. & & & $\mathrm{pI}^{\mathrm{b}}$ & $/ \mathrm{pI}^{\mathrm{c}}$ & erage $(\%)^{\mathrm{e}}$ & $\begin{array}{l}1 \mathrm{~d} \\
/ \mathrm{CK}\end{array}$ & $\begin{array}{l}\mathrm{p} \\
\text { value } \\
\mathrm{g}\end{array}$ & $\begin{array}{l}3 \mathrm{~d} \\
/ \mathrm{CK}\end{array}$ & $\begin{array}{l}\mathrm{p} \\
\text { value } \\
\mathrm{g}\end{array}$ & $\begin{array}{l}5 \mathrm{~d} \\
/ \mathrm{CK}\end{array}$ & $\begin{array}{l}\mathrm{p} \\
\text { value } \\
\mathrm{g}\end{array}$ \\
\hline 97 & GRF1-interacting factor 3 & Q93VH6 & $23.33 / 6.51$ & $\begin{array}{l}23.77 / \\
6.49\end{array}$ & $188 / 17.25$ & 1.37 & 0.04 & 1.74 & 0 & 2.1 & 0.03 \\
\hline 112 & $\begin{array}{l}\text { Transcription initiation factor IIA } \\
\text { subunit } 2\end{array}$ & A2XZI2 & $11.21 / 5.39$ & $\begin{array}{l}11.97 / \\
5.33\end{array}$ & $323 / 48.15 \%$ & 1.12 & 0.01 & 1.55 & 0.03 & 2.18 & 0 \\
\hline \multicolumn{12}{|c|}{ Plant Defense } \\
\hline 1 & Heat shock protein 81 & A2YWQ1 & $82.12 / 4.91$ & $\begin{array}{l}80.19 / \\
5.00\end{array}$ & $112 / 10.97 \%$ & 1.46 & 0.02 & 2.34 & 0.04 & 3.23 & 0.04 \\
\hline 12 & Beta-galactosidase 8 & Q0DGD7 & $81.45 / 5.54$ & $\begin{array}{l}73.52 / \\
5.56\end{array}$ & $115 / 8.76 \%$ & 1.47 & 0.03 & 2.15 & 0.02 & 2.87 & 0.02 \\
\hline 38 & $\begin{array}{l}\text { Light-independent } \\
\text { protochlorophyllide reductase } \\
\text { subunit B. }\end{array}$ & A2T391 & $64.59 / 6.17$ & $\begin{array}{l}52.13 / \\
5.83\end{array}$ & $198 / 16.95 \%$ & 1.59 & 0.03 & 1.91 & 0.02 & 2.11 & 0.02 \\
\hline 54 & Thioredoxin reductase NTRC & Q70G58 & $50.53 / 5.41$ & $\begin{array}{l}51.46 / \\
5.38\end{array}$ & $212 / 19.85 \%$ & 0.71 & 0.03 & 0.46 & 0.02 & 0.24 & 0.02 \\
\hline 57 & Glutathione synthetase & P46416 & $50.81 / 5.64$ & $\begin{array}{l}53.85 / \\
5.53\end{array}$ & $115 / 10.40 \%$ & 1.11 & 0.02 & 1.71 & 0.02 & 2.34 & 0.01 \\
\hline 59 & $\begin{array}{l}\text { Light-independent } \\
\text { protochlorophyllide reductase } \\
\text { subunit B }\end{array}$ & A2T312 & $52.84 / 5.94$ & $\begin{array}{l}57.68 / \\
6.00\end{array}$ & $134 / 8.48 \%$ & 0.66 & 0.03 & 0.38 & 0.01 & 0.21 & 0.01 \\
\hline 61 & Chalcone synthase & O65872 & $45.45 / 6.1$ & $\begin{array}{l}43.20 / \\
6.04\end{array}$ & $166 / 17.66 \%$ & 2.1 & 0.01 & 2.56 & 0.04 & 3.43 & 0.03 \\
\hline
\end{tabular}




\begin{tabular}{|c|c|c|c|c|c|c|c|c|c|c|c|}
\hline Spot & NCBI Accession No. ${ }^{a}$ & Protein Name & Exp.Mw / & Theo. Mw & Score $^{\mathrm{d}} /$ Cov- & & & & tio $^{f}$ & & \\
\hline NO. & & & $\mathrm{pI}^{\mathrm{b}}$ & $/ \mathrm{pI}^{\mathrm{c}}$ & erage $(\%)^{\mathrm{e}}$ & $\begin{array}{l}1 \mathrm{~d} \\
/ \mathrm{CK}\end{array}$ & $\begin{array}{l}\mathrm{p} \\
\text { value } \\
\mathrm{g}\end{array}$ & $\begin{array}{l}3 \mathrm{~d} \\
/ \mathrm{CK}\end{array}$ & $\begin{array}{l}\mathrm{p} \\
\text { value } \\
\mathrm{g}\end{array}$ & $\begin{array}{l}5 \mathrm{~d} \\
/ \mathrm{CK}\end{array}$ & $\begin{array}{l}\mathrm{p} \\
\text { value } \\
\mathrm{g}\end{array}$ \\
\hline 68 & $\begin{array}{l}\text { Guanine nucleotide-binding protein } \\
\text { alpha- } 1 \text { subunit }\end{array}$ & $\mathrm{A} 2 \mathrm{Y} 3 \mathrm{~B} 5$ & $42.40 / 6.80$ & $\begin{array}{l}44.07 / \\
6.71\end{array}$ & $147 / 15.25 \%$ & 1.68 & 0.03 & 2.15 & 0.02 & 2.98 & 0.01 \\
\hline 72 & Glucan endo-1,3-beta-glucosidase & A7PQW3 & $32.43 / 4.76$ & $\begin{array}{l}34.17 / \\
4.75\end{array}$ & $221 / 20.00 \%$ & 1.75 & 0.04 & 2.65 & 0.02 & 2.98 & 0.03 \\
\hline 77 & Cysteine synthase & Q00834 & $31.58 / 5.47$ & $\begin{array}{l}34.18 / \\
5.53\end{array}$ & $205 / 19.94 \%$ & 1.8 & 0.02 & 2.68 & 0.01 & 3.98 & 0.03 \\
\hline 84 & Superoxide dismutase & A39267 & $23.89 / 5.19$ & $\begin{array}{l}25.49 / \\
5.39\end{array}$ & $175 / 16.96 \%$ & 1.25 & 0 & 1.51 & 0.04 & 2.43 & 0.02 \\
\hline 85 & Ascorbate peroxidase & BAA08264 & $25.62 / 5.24$ & $\begin{array}{l}27.16 / \\
5.42\end{array}$ & $159 / 17.79 \%$ & 1.26 & 0.01 & 1.87 & 0.02 & 3.21 & 0.01 \\
\hline 87 & Glutathione S-transferase 1 & P12653 & $23.22 / 5.41$ & $\begin{array}{l}23.69 / \\
5.45\end{array}$ & $223 / 36.23 \%$ & 1.84 & 0.01 & 2.23 & 0.02 & 3.22 & 0.04 \\
\hline 98 & $\begin{array}{l}\text { Desiccation-related protein } \\
\text { At2g46140 }\end{array}$ & O82355 & $16.47 / 4.62$ & $\begin{array}{l}27.23 / \\
6.04\end{array}$ & $233 / 24.80 \%$ & 1.43 & 0.04 & 2.45 & 0.02 & 2.1 & 0 \\
\hline 100 & Salt stress-induced protein & A2WPN7 & $14.79 / 5.11$ & $\begin{array}{l}15.06 / \\
5.19\end{array}$ & $231 / 38.51 \%$ & 1.26 & 0.01 & 2.56 & 0.02 & 2.98 & 0.01 \\
\hline 101 & Thioredoxin H-type & A2YIW7 & $13.84 / 5.22$ & $\begin{array}{l}13.15 / \\
5.17\end{array}$ & $267 / 32.80 \%$ & 1.17 & 0.02 & 1.5 & 0 & 2.54 & 0.01 \\
\hline 102 & $\begin{array}{l}\text { NAD }(\mathrm{P}) \mathrm{H} \text {-quinone oxidoreductase } \\
\text { subunit 3, chloroplastic }\end{array}$ & $\mathrm{A} 2 \mathrm{CI} 37$ & $12.76 / 5.18$ & $\begin{array}{l}13.73 / \\
5.28\end{array}$ & $323 / 41.80 \%$ & 0.72 & 0.01 & 0.43 & 0.03 & 0.11 & 0.03 \\
\hline 106 & 16.6 kDa heat shock protein & Q943Q3 & $\begin{array}{l}\text { pI:6.32, } \\
\text { Mw: } 16649\end{array}$ & $\begin{array}{l}16.71 / \\
6.55\end{array}$ & $204 / 41.18 \%$ & 1.51 & 0.01 & 1.92 & 0.01 & 3.32 & 0.03 \\
\hline
\end{tabular}




\begin{tabular}{|c|c|c|c|c|c|c|c|c|c|c|c|}
\hline Spot & NCBI Accession No. ${ }^{\mathrm{a}}$ & Protein Name & Exp.Mw / & Theo. Mw & Score $/$ Cov- & & & & tio $^{f}$ & & \\
\hline NO. & & & $\mathrm{pI}^{\mathrm{b}}$ & $/ \mathrm{pI}^{\mathrm{c}}$ & erage $(\%)^{\mathrm{e}}$ & $\begin{array}{l}1 \mathrm{~d} \\
/ \mathrm{CK}\end{array}$ & $\begin{array}{l}\mathrm{p} \\
\text { value } \\
\mathrm{g}\end{array}$ & $\begin{array}{l}3 \mathrm{~d} \\
/ \mathrm{CK}\end{array}$ & $\begin{array}{l}\mathrm{p} \\
\text { value } \\
\mathrm{g}\end{array}$ & $\begin{array}{l}5 \mathrm{~d} \\
/ \mathrm{CK}\end{array}$ & $\begin{array}{l}\mathrm{p} \\
\text { value } \\
\mathrm{g}\end{array}$ \\
\hline 115 & $\begin{array}{l}\text { Isoform } 2 \text { of Cysteine proteinase } \\
\text { inhibitor } 1\end{array}$ & P09229-2 & $\begin{array}{l}\text { pI: } 6.40, \\
\text { Mw: } 10068\end{array}$ & 10.84 & $331 / 47.55 \%$ & 1.15 & 0 & 2.17 & 0.01 & 3.54 & 0.02 \\
\hline \multicolumn{12}{|c|}{ Transport protein } \\
\hline 15 & Oligopeptide transporter 7 & O82485 & $56.13 / 5.71$ & $84.8 / 5.84$ & $126 / 11.17 \%$ & 1.17 & 0.04 & 2.82 & 0.03 & 3.45 & 0.02 \\
\hline 56 & Polyamine transporter PUT1 & $\mathrm{A} 2 \mathrm{X} 8 \mathrm{M} 8$ & $56.62 / 5.53$ & $\begin{array}{l}54.25 / \\
5.58\end{array}$ & $98 / 10.19 \%$ & 1.49 & 0.02 & 1.91 & 0.01 & 2.45 & 0.04 \\
\hline 64 & Zinc transporter 8 & A3BI1 1 & $\begin{array}{l}37.80 / \\
6.11\end{array}$ & $41.1 / 6.22$ & $213 / 20.40 \%$ & 1.32 & 0.02 & 1.85 & 0.03 & 2.11 & 0.03 \\
\hline 16 & plasma membrane $\mathrm{H}+$ ATPase & CAD29297 & $91.77 / 6.19$ & $\begin{array}{l}91.20 / \\
6.24\end{array}$ & $115 / 9.80 \%$ & 1.51 & 0 & 2.11 & 0.03 & 3.43 & 0.04 \\
\hline
\end{tabular}

\section{Notes:}

a Database accession numbers according to the Unport/Swiss protein database

b Experimental $M_{\mathrm{w}} / \mathrm{p} I$

c Theoretical $M_{\mathrm{w}} / \mathrm{p} I$

d Mascot search score against the NCBInr database

e Sequences coverage

f Different protein spot intensity ratios after treatment with $300 \mathrm{mM}$ salt for various periods; $1 \mathrm{~d} / \mathrm{CK}$ : the different protein spot intensity ratio after $300 \mathrm{mM}$ salt treatment for $1 \mathrm{~d}$ relative to the control; $3 \mathrm{~d} / \mathrm{CK}$ : the different protein spot intensity ratio after $300 \mathrm{mM}$ salt treatment for $3 \mathrm{~d}$ relative to the control; 5 $\mathrm{d} / \mathrm{CK}$ : the different protein spot intensity ratio after $300 \mathrm{mM}$ salt treatment for $5 \mathrm{~d}$ relative to the control

$\mathrm{g}$ Protein spots showed a significant change in abundance (fold change) by a factor $>1.5$-fold or $<0.6$ fold compared to the control analyzed by an LSD test. A p-value of $<0.05$ was considered statistically significant. 